TRANSACTIONS OF THE

AMERICAN MATHEMATICAL SOCIETY

Volume 311, Number 1, January 1989

\title{
CONJUGATING HOMEOMORPHISMS TO UNIFORM HOMEOMORPHISMS
}

\author{
KATSURO SAKAI AND RAYMOND Y. WONG
}

\begin{abstract}
Let $H(X)$ denote the group of homeomorphisms of a metric space $X$ onto itself. We say that $h \in H(X)$ is conjugate to $g \in H(X)$ if $g=f h f^{-1}$ for some $f \in H(X)$. In this paper, we study the questions: When is $h \in H(X)$ conjugate to $g \in H(X)$ which is a uniform homeomorphism or can be extended to a homeomorphism $\tilde{g}$ on the metric completion of $X$ ? Typically for a complete metric space $X$, we prove that $h \in H(X)$ is conjugate to a uniform homeomorphism if $H$ is uniformly approximated by uniform homeomorphisms. In case $X=\mathbf{R}$, we obtain a stronger result showing that every homeomorphism on $\mathbf{R}$ is, in fact, conjugate to a smooth Lipschitz homeomorphis. For a noncomplete metric space $X$, we provide answers to the existence of $\tilde{g}$ under several different settings. Our results are concerned mainly with infinite-dimensional manifolds.
\end{abstract}

\section{INTRODUCTION}

All spaces considered in this paper are metric spaces. Given a metric space $X=(X, d)$, let $H(X)$ denote the group of homeomorphisms of $X$ onto itself. We say $h \in H(X)$ is conjugate to $g \in H(X)$ if there is an $f \in H(X)$ such that $g=f h f^{-1}$. Let $H_{U}(X)$ denote the set consisting of all $h \in H(X)$ such that both $h$ and $h^{-1}$ are uniformly continuous. Members of $H_{U}(X)$ will be referred to as uniform homeomorphisms. Let $\widetilde{X}$ be the metric completion of $X$. In this paper, we study the following questions:

(A) When is an $h \in H(X)$ conjugate to a $g \in H_{U}(X)$ ?

(B) When is an $h \in H(X)$ conjugate to a $g \in H(X)$ which can be extended to a $\tilde{g} \in H(\tilde{X})$ ?

Question (B) was raised in [AB] (and [AK, CSQ 4]) in the setting of $X=$ $s=(-1,1)^{\infty}$ and $\widetilde{X}=Q=[-1,1]^{\infty}$. Since any $g \in H_{U}(X)$ can be extended to a $\tilde{g} \in H(\tilde{X})$ (in fact, to a $\tilde{g} \in H_{U}(\tilde{X})$ ), (A) implies (B). In general, (B) does not imply (A). However, if $\widetilde{X}$ is compact, then (B) is equivalent to (A).

In $\S 1$, we present the special case $X=\mathbf{R}$, the real line with Euclidean metric. Our result shows that, in fact, every homeomorphism on $\mathbf{R}$ is conjugate to a

Received by the editors March 19, 1987 and, in revised form, October 19, 1987.

1980 Mathematics Subject Classification (1985 Revision). Primary 58D15, 58D05, 57N20.

Key words and phrases. Homeomorphism, uniform homeomorphism, conjugation.

This paper was completed while the first author was visiting the University of California, Santa Barbara. 
smooth Lipschitz homeomorphism with bilip $\leq 1+\varepsilon$ for any given $\varepsilon>0$ (see $\S 1$ for definition).

In $\S 2$, we provide an answer for Question (A) in case $X$ is a complete metric space. Our main result of the section (Theorem 2.1) shows that $h \in H(X)$ is conjugate to a $g \in H_{U}(X)$ provided $h \in \overline{H_{U}(X)}$, that is, $h$ is uniformly approximated by uniform homeomorphisms. As a corollary, we obtain a similar result in case $X$ is the interior of a complete metric $n$-manifold $\widetilde{X}$. In general, we cannot weaken the condition $h \in \overline{H_{U}(X)}$. More specifically, in Example 2.4 we construct a uniformly continuous homeomorphism $h$ on a complete metric, connected 2-manifold $X$ with $\partial X \neq \varnothing$ such that $h$ is not conjugate to any uniform homeomorphism on $X$.

In $\S 3$, we mainly consider finite- or infinite-dimensional manifold cases. Our results answer Question (B) in several different settings. For example, we show that if $X$ is the interior of an $n$-manifold $\widetilde{X}, n \neq 4,5$, and if $h \in H(X)$ extends to a proper map of $\tilde{X}$ then $h$ is conjugate to a $g \in H(X)$ which extends to a $\tilde{g} \in H(\tilde{X})$ (Theorem 3.3). In general, we cannot eliminate the condition that $h$ extends to a proper map of $\tilde{X}$. In Example 3.4 we construct a homeomorphism $h: \dot{B}^{n} \rightarrow \dot{B}^{n}, n \geq 2$, where $\dot{B}^{n}$ is the interior of the $n$-ball $B^{n}$, such that $h$ is not conjugate to any uniform homeomorphism (hence not extendible to $B^{n}$ ).

In $\S 4$, we consider the stable cases for infinite-dimensional manifolds where the given homeomorphisms are of the form (or conjugate to) $h \times$ id. Roughly speading, we show that stable homeomorphisms are always conjugate either to uniform homeomorphisms or to homeomorphisms extendible to its completion.

Finally in $\S 5$, we raise various questions.

\section{LIPSCHITZ CONJUGATION OF HOMEOMORPHISMS OF $\mathbf{R}$}

Let $\left(X_{1}, d_{1}\right)$ and $\left(X_{2}, d_{2}\right)$ be metric spaces. A bijective map $f: X_{1} \rightarrow X_{2}$ is called a Lipschitz homeomorphism if there exists a real number $L \geq 1$ such that

$$
L^{-1} d_{1}(x, y) \leq d_{2}(f(x), f(y)) \leq L d_{1}(x, y)
$$

for all $x, y \in X_{1}$. Let $\operatorname{bilip}(f)$ denote the least such constant $L$ for $f$. Our main result in this section is

1.1. Theorem. Given $\varepsilon>0$ and a homeomorphism $h$ of $\mathbf{R}$ onto itself, then $h$ is conjugate to a smooth Lipschitz homeomorphism $g$ with $\operatorname{bilip}(g) \leq 1+\varepsilon$.

We start with the two special cases: fixed point free homeomorphisms and periodic homeomorphisms.

1.2. Proposition. Any fixed point free homeomorphism of $\mathbf{R}$ is conjugate to the translation $\tau: \mathbf{R} \rightarrow \mathbf{R}$ defined by $\tau(x)=x+1$.

Proof. Let $h \in H(\mathbf{R})$ be fixed point free. Then (1) $h(x)>x$ for all $x \in \mathbf{R}$ or (2) $h(x)<x$ for all $x \in \mathbf{R}$. In case $(1), h^{n}(0) \rightarrow \pm \infty$ as $n \rightarrow \pm \infty$, and in case $(2), h^{n}(0) \rightarrow \mp \infty$ as $n \rightarrow \pm \infty$. Let $f_{0}:[0, h(0)] \rightarrow[0,1]$ (replace 
$[0, h(0)]$ by $[h(0), 0]$ in case (2)) be a homeomorphism such that $f_{0}(0)=0$ and $f_{0}(h(0))=1$. Extend $f_{0}$ to a homeomorphism $f: \mathbf{R} \rightarrow \mathbf{R}$ by

$$
f \mid\left[h^{n}(0), h^{n+1}(0)\right]=\tau^{n} f_{0} h^{-n} \text { for each } n \in \mathbf{Z}
$$

(replace $\left[h^{n}(0), h^{n+1}(0)\right]$ by $\left[h^{n+1}(0), h^{n}(0)\right]$ in case (2)). As easily observed, $f h=\tau f$, so $h$ is conjugate to $\tau$.

Hereafter, let $r: \mathbf{R} \rightarrow \mathbf{R}$ denote the reflection $r(x)=-x$.

1.3. Proposition. Any periodic homeomorphism of $\mathbf{R}$ is conjugate to the reflection $r$.

Proof. Let $h \in H(\mathbf{R})$ be periodic. Then clearly $h^{2}=$ id and $h$ has exactly one fixed point $c \in \mathbf{R}$. Hence $x \leq c$ if and only if $h(x) \geq c$. We define a homeomorphism $f: \mathbf{R} \rightarrow \mathbf{R}$ as follows:

$$
f(x)= \begin{cases}x-c & \text { for } x \geq c \\ c-h(x) & \text { for } x \leq c\end{cases}
$$

We can easily check that $f h(x)=-f(x)=r f(x)$ for each $x \in \mathbf{R}$. Thus $h$ is conjugate to $r$.

If $h \in H(\mathbf{R})$ is decreasing (i.e., order reversing), then $h$ has exactly one fixed point $c \in \mathbf{R}$. Let $f: \mathbf{R} \rightarrow \mathbf{R}$ be the homeomorphism defined as in the above proof and let $g=f h f^{-1}$. Then $g(0)=0$ and $g(x)=-x$ whenever $h^{2} f^{-1}(x)=f^{-1}(x)$. Thus we have proved the following lemma, which will be needed in the proof of Theorem 1.1.

1.4. Lemma. If $h \in H(\mathbf{R})$ is decreasing (i.e., order reversing), then $h$ is conjugate to a $g \in H(\mathbf{R})$ such that $g(0)=0$ and $g(x)=-x$ whenever $x$ is a periodic point of $g$.

We now state several lemmas which will also be needed in the proof of Theorem 1.1. The next lemma follows from the mean value theorem and the inverse function theorem.

1.5. Lemma. A smooth map $g: \mathbf{R} \rightarrow \mathbf{R}$ is a Lipschitz homeomorphism if and only if $g^{\prime}(x)$ and $g^{\prime}(x)^{-1}, x \in \mathbf{R}$, are bounded. Furthermore

$$
\operatorname{bilip}(g)=\max \left\{\sup _{x \in \mathbf{R}}\left|g^{\prime}(x)\right|, \sup _{x \in \mathbf{R}}\left|g^{\prime}(x)\right|^{-1}\right\},
$$

where $g^{\prime}$ is the derivative of $g$.

The proofs of the following two lemmas are straightforward and will be omitted.

1.6. Lemma. Let $\left(a_{i}, b_{i}\right), i=1,2, \cdots<n$, be disjoint open intervals, where $n \leq \infty$ and $-\infty \leq a_{i}<b_{i} \leq \infty$, and let $\varepsilon>0$. For each $i=1,2, \ldots$, we are 
given a smooth Lipschitz homeomorphism $g_{i}: \mathbf{R} \rightarrow \mathbf{R}$ such that $g_{i} \mid \mathbf{R} \backslash\left(a_{i}, b_{i}\right)=$ id and $\operatorname{bilip}\left(g_{i}\right) \leq 1+\varepsilon$. Define $g: \mathbf{R} \rightarrow \mathbf{R}$ by

$$
g(x)= \begin{cases}g_{i}(x) & \text { if } a_{i}<x<b_{i}, i=1,2, \ldots, \\ -x & \text { otherwise. }\end{cases}
$$

Then $g$ is a smooth Lipschitz homeomorphism with $\operatorname{bilip}(g) \leq 1+\varepsilon$.

1.7. Lemma. Let $\left(a_{i}, b_{i}\right), i=1,2, \cdots<n$, be disjoint open intervals in $[0, \infty)$ where $n \leq \infty$ and $0 \leq a_{i}<b_{i} \leq \infty$ and let $\varepsilon>0$. For each $i=$ $1,2, \ldots$, we are given a smooth Lipschitz homeomorphism $g_{i}: \mathbf{R} \rightarrow \mathbf{R}$ such that $g_{i} \mid \mathbf{R} \backslash\left(-b_{i},-a_{i}\right) \cup\left(a_{i}, b_{i}\right)=r$ (i.e., $g_{i}(x)=-x$ if $\left.|x| \notin\left(a_{i}, b_{i}\right)\right)$ and $\operatorname{bilip}\left(g_{i}\right) \leq 1+\varepsilon$. Define $g: \mathbf{R} \rightarrow \mathbf{R}$ by

$$
g(x)= \begin{cases}g_{i}(x) & \text { if } a_{i}<|x|<b_{i}, i=1,2, \ldots, \\ -x & \text { otherwise. }\end{cases}
$$

Then $g$ is a smooth Lipschitz homeomorphism with $\operatorname{bilip}(g) \leq 1+\varepsilon$.

1.8. Lemma. Let $h \in H([a, b]),-\infty \leq a<b \leq \infty$ and $\varepsilon>0$. Suppose $h(x) \neq x$ if and only if $x \neq a, b$. Then there is an $f \in H([a, b])$ and $a$ smooth Lipschitz homeomorphism $g: \mathbf{R} \rightarrow \mathbf{R}$ such that $f(a)=a, f(b)=b$, $g \mid \mathbf{R} \backslash(a, b)=\mathrm{id}, \operatorname{bilip}(g) \leq 1+\varepsilon$ and $g \mid[a, b]=f h f^{-1}$.

Proof. We may assume that $h(x)>x$ for all $x \in(a, b)$ (otherwise replace $h$ by $h^{-1}$ in the argument). Let $k: \mathbf{R} \rightarrow \mathbf{R}$ be a nonnegative smooth map such that $k^{-1}(0)=\mathbf{R} \backslash(a, b)$ and let

$$
\delta=\frac{\varepsilon}{(1+\varepsilon) \cdot \sup _{x \in \mathbf{R}}\left|k^{\prime}(x)\right|}>0 .
$$

Define a smooth map $g: \mathbf{R} \rightarrow \mathbf{R}$ by $g(x)=x+\delta k(x)$. Since $g^{\prime}(x)=1+$ $\delta k^{\prime}(x)$, we have

$$
\frac{1}{1+\varepsilon}=1-\frac{\varepsilon}{1+\varepsilon} \leq 1-\delta\left|k^{\prime}(x)\right| \leq\left|g^{\prime}(x)\right| \leq 1+\delta\left|k^{\prime}(x)\right|<1+\varepsilon
$$

Thus $g$ is a smooth Lipschitz homeomorphism with bilip $(g) \leq 1+\varepsilon$ by Lemma 1.5. Note that $g(x)>x$ for all $x \in(a, b)$. We shall show that $h$ is conjugate to $g \mid[a, b]$. To this end, let $c \in(a, b)$. We can easily verify that $h^{n}(c) \rightarrow a$ and $g^{n}(c) \rightarrow a$ as $n \rightarrow-\infty$, and also $h^{n}(c) \rightarrow b$ and $g^{n}(c) \rightarrow b$ as $n \rightarrow$ $\infty$. Let $f_{0}:[c, h(c)] \rightarrow[c, g(c)]$ be a homeomorphism such that $f_{0}(c)=c$ and $f_{0}(h(c))=g(c)$. Extend $f_{0}$ to a homeomorphism $f:[a, b] \rightarrow[a, b]$ by $f(a)=a, f(b)=b$ and

$$
f \mid\left[h^{n}(c), h^{n+1}(c)\right]=g^{n} f_{0} h^{-n} \quad \text { for each } n \in \mathbf{Z} .
$$

Then $f h=g f$; hence $h$ is conjugate to $g \mid[a, b]$.

1.9. Lemma. Let $h \in H([-b,-a] \cup[a, b]), 0 \leq a<b \leq \infty$ and $\varepsilon>0$. Suppose $h( \pm a)=\mp a, h( \pm b)=\mp b$ and $h$ has no point of period 2 except $\pm a$ and 
$\pm b$. Then there is an $f \in H([-b,-a] \cup[a, b])$ and a smooth Lipschitz homeomorphism $g: \mathbf{R} \rightarrow \mathbf{R}$ such that $f( \pm a)= \pm a, f( \pm b)= \pm b, g \mid \mathbf{R} \backslash((-b,-a) \cup$ $(a, b))=r$ (the reflection), $\operatorname{bilip}(g) \leq 1+\varepsilon$ and $g \mid[-b,-a] \cup[a, b]=f h f^{-1}$.

Proof. We may assume that $h^{2}(x)>x$ for all $x \in(a, b)$ (otherwise replace $h^{2}$ by $h^{-2}$ in the argument). It follows that $h^{2}(x)<x$ for all $x \in(-b,-a)$. Let $k: \mathbf{R} \rightarrow \mathbf{R}$ and $\delta>0$ be as in the proof of Lemma 1.8. Define a smooth map $g: \mathbf{R} \rightarrow \mathbf{R}$ by

$$
g(x)= \begin{cases}-x-\delta k(x) & \text { for } x \geq 0 \\ -x+\delta k(-x) & \text { for } x \leq 0\end{cases}
$$

Since $g^{\prime}(x)=-1-\delta k^{\prime}(|x|)$, we have

$$
\frac{1}{1+\varepsilon}=1-\frac{\varepsilon}{1+\varepsilon} \leq 1-\delta\left|k^{\prime}(x)\right| \leq\left|g^{\prime}(x)\right| \leq 1+\delta\left|k^{\prime}(x)\right|<1+\varepsilon .
$$

Then $g$ is a smooth Lipschitz homeomorphism with bilip $(g) \leq 1+\varepsilon$ by Lemma 1.5. Note that $g( \pm a)=\mp a, g( \pm b)=\mp b$ and $g^{2}(x)>x$ for all $x \in(a, b)$ (hence $g^{2}(x)<x$ for all $x \in(-b,-a)$ ).

We want to show that $h$ is conjugate to $g \mid[-b,-a] \cup[a, b]$. To this end, let $c \in(a, b)$. Observe that $h^{2 n}(c) \rightarrow a$ and $g^{2 n}(c) \rightarrow a$ as $n \rightarrow-\infty ; h^{2 n}(c) \rightarrow b$ and $g^{2 n}(c) \rightarrow b$ as $n \rightarrow \infty ; h^{2 n+1}(c) \rightarrow-a$ and $g^{2 n+1}(c) \rightarrow-a$ as $n \rightarrow-\infty$; and $h^{2 n+1}(c) \rightarrow-b$ and $g^{2 n+1}(c) \rightarrow-b$ as $n \rightarrow \infty$. Let $f_{0}:\left[c, h^{2}(c)\right] \rightarrow$ $\left[c, g^{2}(c)\right]$ be a homeomorphism such that $f_{0}(c)=c$ and $f_{0}\left(h^{2}(c)\right)=g^{2}(c)$. Extend $f_{0}$ to a homeomorphism $f:[-b,-a] \cup[a, b] \rightarrow[-b,-a] \cup[a, b]$ by $f( \pm a)= \pm a, f( \pm b)= \pm b$ and

$$
f \mid\left[h^{n}(c), h^{n+2}(c)\right]=g^{n} f_{0} h^{-n} \text { for each } n \in \mathbf{Z} .
$$

Then $f h=g f$; hence $h$ is conjugate to $g \mid[-b,-a] \cup[a, b]$.

Proof of Theorem 1.1. Let $h \in H(\mathbf{R})$ and $\varepsilon>0$. First we assume that $h$ is increasing (i.e., order preserving). Let Fix $h$ denote the fixed point set of $h$. The case Fix $h=\varnothing$ is Proposition 1.2. Note that $\mathbf{R} \backslash$ Fix $h$ is the disjoint union of open intervals $\left(a_{i}, b_{i}\right), i=1, \ldots, n$, where $n \leq \infty$ and $-\infty \leq a_{i}<b_{i} \leq \infty$. For each $i$, let $h_{i}=h \mid\left[a_{i}, b_{i}\right] \in H\left(\left[a_{i}, b_{i}\right]\right)$ and $\varepsilon_{i}=\varepsilon$ for each $i$. By Lemma 1.8, we have a smooth Lipschitz homeomorphism $g_{i}: \mathbf{R} \rightarrow \mathbf{R}$ and $f_{i} \in H\left(\left[a_{i}, b_{i}\right]\right)$ such that $g_{i} \mid \mathbf{R} \backslash\left(a_{i}, b_{i}\right)=\mathrm{id}$, $\operatorname{bilip}\left(g_{i}\right) \leq 1+\varepsilon_{i}, f_{i}\left(a_{i}\right)=a_{i}$, $f_{i}\left(b_{i}\right)=b_{i}$ and $g_{i} \mid\left[a_{i}, b_{i}\right]=f_{i} h_{i} f_{i}^{-1}$. Define $f, g: \mathbf{R} \rightarrow \mathbf{R}$ by $f \mid$ Fix $h=$ $g \mid$ Fix $h=\mathrm{id}, f \mid\left[a_{i}, b_{i}\right]=f_{i}$ and $g \mid\left[a_{i}, b_{i}\right]=g_{i}$ for each $i$. Then $f$ is clearly a homeomorphism and $g$ is a smooth Lipschitz homeomorphism by Lemma 1.6. Since $g=f h f^{-1}, h$ is conjugate to $g$.

Next we assume that $h$ is decreasing (i.e., order reversing). By Lemma 1.4, we may assume that $h(0)=0, h(x)=-x$ if $x$ is a periodic point of $h$. Let Per $h$ denote the periodic point set of $h$. The case $\operatorname{Per} h=\mathbf{R}$ is Proposition 1.3. Note that $[0, \infty) \backslash \operatorname{Per} h$ is the disjoint union of open intervals $\left(a_{i}, b_{i}\right)$, 
$i=1,2, \ldots, n$, where $n \leq \infty$ and $0 \leq a_{i}<b_{i} \leq \infty$, and $(-\infty, 0] \backslash \operatorname{Per} h$ is the disjoint union of

$$
\left(-b_{i},-a_{i}\right)=h\left(\left(a_{i}, b_{i}\right)\right), \quad i=1,2, \ldots, n .
$$

Let $h_{i}=h \mid\left[-b_{i},-a_{i}\right] \cup\left[a_{i}, b_{i}\right] \in H\left(\left[-b_{i},-a_{i}\right] \cup\left[a_{i}, b_{i}\right]\right)$ and $\varepsilon_{i}=\varepsilon$ for each $i$. By Lemma 1.9, we have a smooth Lipschitz homeomorphism $g_{i}: \mathbf{R} \rightarrow \mathbf{R}$ and $f_{i} \in H\left(\left[-b_{i},-a_{i}\right] \cup\left[a_{i}, b_{i}\right]\right)$ such that $g \mid \mathbf{R} \backslash\left(\left(-b_{i},-a_{i}\right) \cup\left(a_{i}, b_{i}\right)\right)=r$, $\operatorname{bilip}\left(g_{i}\right) \leq 1+\varepsilon_{i}, f_{i}\left( \pm a_{i}\right)= \pm a_{i}, f_{i}\left( \pm b_{i}\right)= \pm b_{i}$ and $g_{i} \mid\left[-b_{i},-a_{i}\right] \cup\left[a_{i}, b_{i}\right]=$ $f_{i} h_{i} f_{i}^{-1}$. Define $f, g: \mathbf{R} \rightarrow \mathbf{R}$ by $f|\operatorname{Per} h=\mathrm{id}, g| \operatorname{Per} h=r, f \mid\left[-b_{i},-a_{i}\right] \cup$ $\left[a_{i}, b_{i}\right]=f_{i}$ and $g \mid\left[-b_{i},-a_{i}\right] \cup\left[a_{i}, b_{i}\right]=g_{i}$ for each $i$. Then $f$ is clearly a homeomorphism and $g$ is a smooth Lipschitz homeomorphism by Lemma 1.7. Since $g=f h f^{-1}, h$ is conjugate to $g$.

Remark. One should remark that Theorem 1.1 does not hold for $(0, \infty)$. In fact, any decreasing (order reversing) homeomorphism of $(0, \infty)$ (e.g., $x \rightarrow$ $1 / x)$ is not conjugate to any uniform homeomorphism.

\section{THE CONJUGATION PROBLEM (A): COMPLETE METRIC CASE}

For a given metric space $X=(X, d)$, let $d_{1}$ be the metric on $X$ defined by $d_{1}(x, y)=\min \{d(x, y), 1\}$. Then $d_{1}$ is uniformly equivalent to $d$. Replacing $d$ by $d_{1}$, we may assume without loss of generality that the metric $d$ of $X$ is bounded by 1. Let $C(X)$ denote the space of maps of $X$ into itself with the sup-metric $d^{*}$ defined by

$$
d^{*}(f, g)=\sup \{d(f(x), g(x)) \mid x \in X\} .
$$

Let $C_{U}(X)$ denote the subset consisting of uniformly continuous maps. Then $C_{U}(X)$ is closed in $C(X)$; hence $\overline{H_{U}(X)} \subset C_{U}(X)$ where $\overline{H_{U}(X)}$ is the closure of $H_{U}(X)$ in $C(X)$. Our main result in this section is the following theorem.

2.1. Theorem. Let $(X, d)$ be a complete metric space. Then each $h \in H(X) \cap$ $\overline{H_{U}(X)}$ is conjugate to a $g \in H_{U}(X)$.

Proof. For each $i \in \mathbf{Z}$ (= integers), let $X_{i}$ denote a copy of $X$ and let $X^{\mathbf{Z}}=$ $\prod_{i \in \mathbf{Z}} X_{i}$ with the product metric $\rho$ defined by

$$
\rho\left(\left(x_{i}\right)_{i \in \mathbf{Z}},\left(y_{i}\right)_{i \in \mathbf{Z}}\right)=\sup \left\{2^{-|i|} \cdot d\left(x_{i}, y_{i}\right) \mid i \in \mathbf{Z}\right\} .
$$

Let $\pi_{i}: X^{\mathbf{Z}} \rightarrow X_{i}=X, i \in \mathbf{Z}$, be the projections and $S: X^{\mathbf{Z}} \rightarrow X^{\mathbf{Z}}$ be the left-shift homeomorphism of $X^{Z}$ defined by $\pi_{i} \circ S=\pi_{i+1}$, that is,

$$
S\left(\ldots, x_{-2}, x_{-1}, x_{0}, x_{1}, x_{2}, \ldots\right)=\left(\ldots, x_{-1}, x_{0}, x_{1}, x_{2}, x_{3}, \ldots\right) .
$$

Then $S \in H_{U}\left(X^{\mathbf{Z}}\right)$. We define the infinite graph of $h$ as follows:

$$
X_{h}=\left\{\left(x_{i}\right)_{i \in \mathbf{Z}} \in X^{\mathbf{Z}} \mid x_{n}=h^{n}\left(x_{0}\right) \text { for } n \neq 0\right\} .
$$


Then clearly $S\left(X_{h}\right)=X_{h}$ and $\pi=\pi_{0} \mid X_{h}: X_{h} \rightarrow X$ is a homeomorphism of $X_{h}$ onto $X$. Thus we have a commutative diagram:

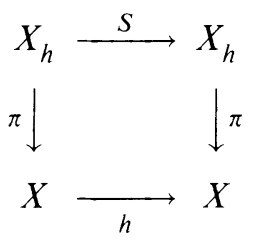

Let $X_{\infty}=\lim _{\longleftarrow}\left(X_{i}, h_{i}\right) \subset \prod_{i=0}^{\infty} X_{i}$ denote the inverse limit of $h_{i}=h: X_{i} \rightarrow$ $X_{i-1}, i=1,2, \ldots$, that is,

$$
\begin{aligned}
X_{\infty} & =\left\{\left(x_{0}, x_{1}, \ldots\right) \mid h\left(x_{n}\right)=x_{n-1} \text { for each } n\right\} \\
& =\left\{\left(x_{0}, x_{1}, \ldots\right) \mid x_{n}=h^{-n}\left(x_{0}\right) \text { for } n>0\right\} .
\end{aligned}
$$

The metric of $X_{\infty}$ is the product metric defined similarly as the metric $\rho$ of $X^{\mathbf{Z}}$. Define $q: X_{h} \rightarrow X_{\infty}$ by

$$
q\left(\ldots, x_{-2}, x_{-1}, x_{0}, x_{1}, x_{2}, \ldots\right)=\left(x_{0}, x_{-1}, x_{-2}, \ldots\right) .
$$

Obviously $q$ is uniformly continuous. Since the size of $X_{n}$ is uniformly small for large $n$ (under the product metric), uniform continuity of each $h^{n}$ implies uniform continuity of $q^{-1}$. Hence $q$ is a uniform homeomorphism.

We want to show that $X_{\infty}$ is uniformly homeomorphic to $X$. By hypothesis, each bonding map $h_{i}: X_{i} \rightarrow X_{i-1}$ can be approximated (in the sup-metric $d^{*}$ of $C(X))$ by uniform homeomorphisms. In the following construction, we employ a procedure of $\mathrm{M}$. Brown [Br] ( $\mathrm{cf}$. $\left[\mathrm{Cu}_{1}\right.$, Theorem 3.1]) showing that two inverse limits of compacta are homeomorphic:

Given $\varepsilon_{0}=2^{-1}$, we can choose a uniform homeomorphism $g_{1}: X_{1} \rightarrow X_{0}$ and $0<\varepsilon_{1}<2^{-2}$ so that $d^{*}\left(g_{1}, h_{1}\right)<\varepsilon_{0}$ and for all $x, y \in X_{1}, d(x, y)<$ $2 \varepsilon_{1}$ implies $d\left(h_{1}(x), h_{1}(y)\right)<\varepsilon_{0}$ and $d\left(g_{1}(x), g_{1}(y)\right)<\varepsilon_{0}$. Inductively given uniform homeomorphisms $g_{i}: X_{i} \rightarrow X_{i-1}$ and $0<\varepsilon_{i}<2^{-(i+1)}$ for $i=1, \ldots, n-1$, we can choose a uniform homeomorphism $g_{n}: X_{n} \rightarrow X_{n-1}$ and $0<\varepsilon_{n}<2^{-(n+1)}$ so that $d^{*}\left(g_{n}, h_{n}\right)<\varepsilon_{n-1}$ and for all $x, y \in X_{n}$, $d(x, y)<2 \varepsilon_{n}$ implies $d\left(h_{i n}(x), h_{i n}(y)\right)<\varepsilon_{n-1}$ and $d\left(g_{i n}(x), g_{i n}(y)\right)<\varepsilon_{n-1}$ for each $i=0,1, \ldots, n-1$, where $h_{i n}=h_{i+1} \circ h_{i+1 n}$ and $g_{i n}=g_{i+1} \circ g_{i+1 n}$ $\left(h_{n n}=g_{n n}=\mathrm{id}\right)$. Thus we obtain uniform homeomorphisms $g_{n}: X_{n} \rightarrow X_{n-1}$, $n=1,2, \ldots$. By the choice of $\varepsilon_{n}$, it is straightforward to verify that for all $0 \leq i<j<k$,

$$
d^{*}\left(g_{i j} h_{j k}, g_{i k}\right)<2^{-j} \text { and } d^{*}\left(h_{i j} g_{j k}, h_{i k}\right)<2^{-j} .
$$

First we will show that $X_{\infty}$ is uniformly homeomorphic to

$$
X_{\infty}^{\prime}=\lim _{(}\left(X_{i}, g_{i}\right) \text {. }
$$

Let $h_{i \infty}: X_{\infty} \rightarrow X_{i}$ and $g_{i \infty}: X_{\infty}^{\prime} \rightarrow X_{i}$ be projections. From $(*),\left\{g_{i j} h_{j \infty}\right\}_{j>1}$ is a uniform Cauchy sequence of uniformly continuous maps from $X_{\infty}$ to $X_{i}$. 
Then we can define a uniformly continuous map $\varphi: X_{\infty} \rightarrow X_{\infty}^{\prime}$ by $g_{i \infty} \varphi=$ $\lim _{j \rightarrow \infty} g_{i j} h_{j \infty}$. Likewise we have a uniformly continuous map $\psi: X_{\infty}^{\prime} \rightarrow X_{\infty}$ defined by $h_{i \infty} \psi=\lim _{j \rightarrow \infty} h_{i j} g_{j \infty}$. From $(*)$, it follows that

$$
h_{i \infty} \psi \varphi=\lim _{\substack{j, k \rightarrow \infty \\ i<j<k}} h_{i j} g_{j k} h_{k \infty}=h_{i \infty} ;
$$

hence $\psi \varphi=\mathrm{id}$. Similarly $\varphi \psi=\mathrm{id}$. Therefore $\varphi$ is a uniform homeomorphism. Now $g_{0 \infty}: X_{\infty}^{\prime} \rightarrow X_{0}=X$ is clearly a homeomorphism which is uniformly continuous. Since each $g_{i}^{-1}$ is uniformly continuous, so is $g_{0 \infty}^{-1}$. Hence $g_{0 \infty}$ is also a uniform homeomorphism.

Thus we obtain a uniform homeomorphism $f=g_{0 \infty} \varphi q: X_{h} \rightarrow X$. Then $h$ is conjugate to $g=f S f^{-1} \in H_{U}(X)$ since $g=f \pi^{-1} h \pi f^{-1}$.

Let $(X, d)$ be any metric space (complete or not) and let $h \in H(X)$. In the above proof, we constructed the infinite graph $\left(X_{h}, \rho\right)$ of $h$ and the homeomorphism $\pi: X_{h} \rightarrow X$ such that $S=\pi^{-1} h \pi$ is the left-shift homeomorphism. Define a metric $d_{h}$ on $X$ by $d_{h}(x, y)=\rho\left(\pi^{-1}(x), \pi^{-1}(y)\right)$. Then $d_{h}$ is equivalent to $d$. Since $S$ is in fact a Lipschitz homeomorphism, we have proved

2.2. Corollary. Given any homeomorphism $h$ of a metric space $X$ onto itself, there is an equivalent metric on $X$ making $h$ a Lipschitz homeomorphism.

Let $(X, d)$ be any noncomplete metric space and let $h \in H(X) \cap \overline{H_{U}(X)}$. Since $h$ is uniformly continuous, $h$ extends to a map $\tilde{h}: \widetilde{X} \rightarrow \widetilde{X}$ of the completion of $(X, d)$. Then obviously $\tilde{h} \in \overline{H_{U}(\widetilde{X})}$. Let $\bar{X}_{h}$ be the closure of the infinite graph $X_{h}$ of $h$ in $\widetilde{X}^{\mathbf{Z}}=\prod_{i \in \mathbf{Z}} \widetilde{X}_{i}$. It is easy to see

$$
\bar{X}_{h}=\left\{\left(x_{i}\right)_{i \in \mathbf{Z}} \in \tilde{X}^{\mathbf{Z}} \mid x_{i}=\tilde{h}\left(x_{i-1}\right) \text { for all } i \in \mathbf{Z}\right\} .
$$

By the same arguments as in the proof of Theorem 2.1, we have a uniform homeomorphism $\tilde{f}: \bar{X}_{h} \rightarrow \tilde{X}$. In general, we do not know whether the pair $\left(\bar{X}_{h}, X_{h}\right)$ is uniformly homeomorphic to $(\widetilde{X}, X)$. In case $X$ is the interior of a complete metric $n$-manifold $\tilde{X}, f=\tilde{f} \mid X_{h}: X_{h} \rightarrow X$ is a uniform homeomorphism by the invariance of domain. (In fact, $X_{h}$ is the interior of the manifold $\overline{X_{h}}$ by Lemma 3.2(1).) Thus we have

2.3. Corollary. Let $X$ be the interior of a complete metric n-manifold $\tilde{X}$. Then each $h \in H(X) \cap \overline{H_{U}(X)}$ is conjugate to a $g \in H_{U}(X)$ (which extends to a uniform homeomorphism of $\widetilde{X}$ ).

We conclude this section by exhibiting an example $h \in H(X) \cap C_{U}(X)$ for a connected complete metric 2-manifold $X \subset \mathbf{R}^{2}$ with boundary $\partial X \neq \varnothing$ such that $h$ is not conjugate to any uniform homeomorphism of $X$. 
2.4. Example. Define $a_{n}, n \in \mathbf{Z}$, as follows: $a_{n}=1$ for $n<0, a_{0}=2$ and $a_{n}=\left(a_{n-1}+1\right)^{2}$ for $n>0$. For each $n \in \mathbf{Z}$, we denote the rectangular 2-cell $[2 n, 2 n+1] \times\left[0, a_{n}\right]$ by $D_{n}$ and its boundary and interior by $\partial D_{n}$ and $\dot{D}_{n}$, respectively. Let $X=\mathbf{R}^{2} \backslash \bigcup_{n \in \mathbf{Z}} \dot{D}_{n}$. Then $X$ is a complete metric connected 2manifold with boundary $\partial X=\bigcup_{n \in \mathbf{Z}} \partial D_{n}$. Let $h: X \rightarrow X$ be the canonical p.l. homeomorphism which carries each $\partial D_{n}$ to $\partial D_{n-1}$. Then $h \in H(X) \cap C_{U}(X)$. For each $f \in H(X)$ and each $n \in \mathbf{Z}$, we denote $f\left(\partial D_{n}\right)=\partial D_{f_{*}(n)}$. Then $h_{*}(n)=n-1$.

We want to show that $h$ is not conjugate to any $g \in H_{U}(X)$. So suppose the contrary, that $h$ is conjugate to a $g \in H_{U}(X)$. Then as is easily observed, $g_{*}(n) \neq n$ for any $n \in \mathbf{Z}$. From uniform continuity of $g$ and $g^{-1}$, there is an integer $m>0$ such that for $A \subset X, \operatorname{diam} A \leq 1 / m$ implies $\operatorname{diam} g(A) \leq 1$ and $\operatorname{diam} g^{-1}(A) \leq 1$. Choose an integer $n>0$ so that $a_{n-1} \geq 2 m$. Then $g_{*}(n)<n$ or $g_{*}(n)>n$.

Case 1: $g_{*}(n)<n$. Decompose $\partial D_{g_{*}(n)}$ into $2 m\left(a_{g_{*}(n)}+1\right)$ segments $A_{i}$ with length $=1 / m$, that is, $\partial D_{g_{*}(n)}=\bigcup\left\{A_{i} \mid i=1, \ldots, 2 m\left(a_{g_{*}(n)}+1\right)\right\}$. Then

$$
\begin{aligned}
\operatorname{diam} g^{-1}\left(\partial D_{g_{*}(n)}\right) & \leq \sum\left\{\operatorname{diam} g^{-1}\left(A_{i}\right) \mid i=1, \ldots, 2 m\left(a_{g_{*}(n)}+1\right)\right\} \\
& \leq 2 m\left(a_{g_{*}(n)}+1\right)<\left(a_{n-1}+1\right)^{2}=a_{n} \\
& <\operatorname{diam} \partial D_{n}=\operatorname{diam} g^{-1}\left(\partial D_{g_{*}(n)}\right) .
\end{aligned}
$$

This is a contradiction.

Case 2: $g_{*}(n)>n$. We can similarly write

$$
\partial D_{n}=\bigcup\left\{A_{i} \mid i=1, \ldots, 2 m\left(a_{n}+1\right)\right\},
$$

where each $A_{i}$ is a segment with length $=1 / \mathrm{m}$. Then

$$
\begin{aligned}
\operatorname{diam} g\left(\partial D_{n}\right) & \leq \sum\left\{\operatorname{diam} g\left(A_{i}\right) \mid i=1, \ldots, 2 m\left(a_{n}+1\right)\right\} \\
& \leq 2 m\left(a_{n}+1\right)<\left(a_{n}+1\right)^{2}=a_{n+1} \leq a_{g_{*}(n)} \\
& <\operatorname{diam} \partial D_{g_{*}(n)}=\operatorname{diam} g\left(\partial D_{n}\right) .
\end{aligned}
$$

This is also a contradiction.

\section{The CONJUgation PRoblem (B): MANifold CASE}

Let $X$ and $Y$ be spaces and $\mathscr{U}$ an open cover of $Y$. We say that maps $f, g: X \rightarrow Y$ are $\mathscr{U}$-close if for each $x$ there is a $U \in \mathscr{U}$ such that

$$
\{f(x), g(x)\} \subset U \text {. }
$$

Maps $f, g: X \rightarrow Y$ are $\mathscr{U}$-homotopic if there is a homotopy $h: X \times I \rightarrow Y$ such that $h_{0}=f, h_{1}=g$ and each $h(\{x\} \times I)$ is contained in some member 
of $\mathscr{U}$. A map $f: X \rightarrow Y$ is called a near homeomorphism if for each open cover $\mathscr{U}$ of $Y$ there is a homeomorphism $g: X \rightarrow Y$ which is $\mathscr{U}$-close to $f$. A map $f: X \rightarrow Y$ is called a fine homotopy equivalence if for each open cover $\mathscr{U}$ of $Y$ there is a map $g: Y \rightarrow X$ such that $f g$ is $\mathscr{U}$-homotopic to id and $g f$ is $f^{-1}(\mathscr{U})$-homotopic to id. The proof of the following lemma is rather trivial and will be omitted.

3.1. Lemma. Let $f: X \rightarrow Y$ be a fine homotopy equivalence. Then a map $g: Y \rightarrow Z$ is a fine homotopy equivalence if and only if $g f: X \rightarrow Z$ is a fine homotopy equivalence.

We say that a subset $Y$ of a metric space $X=(X, d)$ is map-dense in $X$ if for any compactum $A \subset X$ and any $\varepsilon>0$, there is a map $f: A \rightarrow Y$ with $d^{*}(f, \mathrm{id})<\varepsilon\left[\mathrm{Cu}_{2}\right]$. A closed subset $K$ of $X$ is called a $Z$-set in $X$ if for any open cover $\mathscr{U}$ of $X$ there is a map $f: X \rightarrow X \backslash K$ with is $\mathscr{U}$-close to id (cf. $\left[\mathrm{Ch}_{2}\right]$ ). In case $X$ is an ANR, this is equivalent to the condition: $X \backslash K$ is map-dense in $X$.

Throughout this section, we will use the following convention. Given a metric space $X=(X, d)$, let $\widetilde{X}$ denote the completion of $X$ and $X^{*}=\tilde{X} \backslash X$. To simplify notation, the metric of $\widetilde{X}$ will also be denoted by $d$. As mentioned before, we may assume that $d$ is bounded by 1 . For each $i \in \mathbf{Z}$, let $\tilde{X}_{i}$ denote a copy of $\tilde{X}$ and let $\tilde{X}^{\mathbf{Z}}=\prod_{i \in \mathbf{Z}} \tilde{X}_{i}$ with the product metric $\rho$ defined as in the proof of Theorem 2.1. Let $\pi_{i}: \widetilde{X}^{\mathbf{Z}} \rightarrow \tilde{X}_{i}=\tilde{X}, i \in \mathbf{Z}$, denote the projections and $S: \tilde{X}^{\mathbf{Z}} \rightarrow \widetilde{X}^{\mathbf{Z}}$ be the left-shift homeomorphism defined by $\pi_{i} S=\pi_{i+1}$. Let $X_{h}$ be the infinite graph of $h \in H(X)$ in $\tilde{X}^{\mathbf{Z}}$, that is,

$$
X_{h}=\left\{\left(x_{i}\right)_{i \in \mathbf{Z}} \in \tilde{X}^{\mathbf{Z}} \mid x_{0} \in X, x_{n} \in h^{n}\left(x_{0}\right) \text { for } n \neq 0\right\},
$$

$\bar{X}_{h}$ the closure of $X_{h}$ in $\tilde{X}^{\mathbf{Z}}$ and $X_{h}^{*}=\bar{X}_{h} \backslash X_{h}$. Then $S\left(X_{h}\right)=X_{h}, S\left(\bar{X}_{h}\right)=$ $\bar{X}_{h}, S\left(X_{h}^{*}\right)=X_{h}^{*}, \pi_{0}\left(X_{h}\right)=X, \pi_{0}\left(\bar{X}_{h}\right)=\tilde{X}, \pi_{0}\left(X_{h}^{*}\right)=X^{*}$ and $\pi=$ $\pi_{0} \mid X_{h}: X_{h} \rightarrow X$ is a homeomorphism. We have the following commutative diagram:

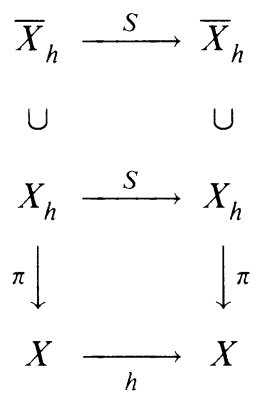

Thus the conjugation problem (B) is equivalent to the problem of whether the pair $\left(\bar{X}_{h}, X_{h}\right)$ is homeomorphic $(\cong)$ to $(\widetilde{X}, X)$.

3.2. Lemma. In the above diagram, suppose that $h$ extends to a map $\tilde{h}: \tilde{X} \rightarrow$ $\tilde{X}$. Then (1) if $X$ is map-dense in $\widetilde{X}$, then $X_{h}$ is also map-dense in $\bar{X}_{h}$, 
(2) if $\tilde{h}$ is proper, then so is $\bar{\pi}=\pi_{0} \mid \bar{X}_{h}: \bar{X}_{h} \rightarrow \tilde{X}$, and (3) if $\tilde{h}$ is a near homeomorphism, then $\bar{\pi}$ is a near homeomorphism.

Proof. First observe

$$
\bar{X}_{h}=\left\{\left(x_{i}\right)_{i \in \mathbf{Z}} \in \tilde{X}^{\mathbf{Z}} \mid x_{i}=\tilde{h}\left(x_{i-1}\right) \text { for all } i \in \mathbf{Z}\right\} .
$$

Let $\tilde{X}_{\infty}=\lim _{(}\left(\tilde{X}_{i}, \tilde{h}_{i}\right)$, where $\tilde{h}_{i}=\tilde{h}: \tilde{X}_{i} \rightarrow \tilde{X}_{i-1}$. Then we have a homeomorphism $q: \bar{X}_{h} \rightarrow \widetilde{X}_{\infty}$ defined by

$$
q\left(\ldots, x_{-2}, x_{-1}, x_{0}, x_{1}, x_{2}, \ldots\right)=\left(x_{0}, x_{-1}, x_{-2}, \ldots\right) .
$$

If $\tilde{h}$ is a near homeomorphism, then the projection $\tilde{h}_{0 \infty}: \tilde{X}_{\infty} \rightarrow \tilde{X}_{0}=\tilde{X}$ is a near homeomorphism by [ $\mathrm{Cu}_{1}$, Theorem 3.3]. (This is proved by the same arguments as Theorem 2.1 using open covers $\mathscr{U}_{i}$ of $\tilde{X}_{i}$ instead of $\varepsilon_{i}>0$ where $\mathscr{U}_{0}$ can be chosen arbitrarily small.) Hence $\bar{\pi}=\tilde{h}_{0 \infty} q: \bar{X}_{h} \rightarrow \widetilde{X}_{0}=\widetilde{X}$ is a near homeomorphism. If $\tilde{h}$ is proper, then $\tilde{h}_{0 \infty}: \tilde{X}_{\infty} \rightarrow \tilde{X}$ is also proper because each bonding map $\tilde{h}_{i}$ is proper. Hence $\bar{\pi}=\tilde{h}_{0 \infty} q$ is proper. Thus we have (3) and (2).

Finally we will prove (1). Let $A$ be a compactum in $\bar{X}_{h}$ and $\varepsilon>0$. Choose an integer $n>0$ so that $2^{-n}<\varepsilon$. Since $\pi_{-n}(A)$ is compact, there is a $\delta>0$ such that if $a \in \pi_{-n}(A), x \in \widetilde{X}$ and $d(a, x)<\delta$, then $d\left(h^{i}(a), h^{i}(x)\right)<\varepsilon$ for $i=0,1, \ldots, 2 n$. Since $X$ is map-dense in $\tilde{X}$, there is a map $f: \pi_{-n}(A) \rightarrow X$ with $d^{*}(f$, id $)<\delta$. Since $\pi_{-n} \mid X_{h}: X_{h} \rightarrow X$ is a homeomorphism, we have a map $g=\left(\pi_{-n} \mid X_{h}\right)^{-1} \circ f \circ\left(\pi_{-n} \mid A\right): A \rightarrow X_{h}$. For each $x=\left(x_{i}\right)_{i \in \mathbf{Z}} \in A$ let $g(x)=\left(y_{i}\right)_{i \in \mathbf{Z}}$. Then $y_{i}=h^{i+n} f\left(x_{-n}\right)$ and $x_{i}=h^{i+n}\left(x_{-n}\right)$ for all $i \in \mathbf{Z}$. Since $d\left(f\left(x_{-n}\right), x_{-n}\right)<\delta$ and $x_{-n} \in \pi_{n}(A)$,

$$
2^{-|i|} d\left(y_{i}, x_{i}\right) \leq d\left(h^{i+n} f\left(x_{-n}\right), h^{i+n}\left(x_{-n}\right)\right)<\varepsilon
$$

provided $|i| \leq n$. Finally if $|i|>n$ then $2^{-|i|} d\left(y_{i}, x_{i}\right)<2^{-n}<\varepsilon$. Therefore $\rho(g(x), x)<\varepsilon$ for each $x \in A$. So $X_{h}$ is map-dense in $\bar{X}_{h}$.

Now we give a version of Corollary 2.3.

3.3. Theorem. Let $n \neq 4,5$ and $X$ be the interior of an $n$-manifold $\widetilde{X}$. If $h \in H(X)$ extends to a proper map $\tilde{h}$ of $\tilde{X}$, then $h$ is conjugate to a $g \in H(X)$ which extends to $\tilde{g} \in H(\widetilde{X})$.

Proof. Since the boundary $\partial \widetilde{X}=X^{*}$ of $\tilde{X}$ has a collar in $\tilde{X}$, the inclusion $X \subset \tilde{X}$ is a fine homotopy equivalence. By Lemma 3.1, $\tilde{h}: \widetilde{X} \rightarrow \widetilde{X}$ is also a fine homotopy equivalence, hence a CE-map. By the result of Siebenmann [Si], $\tilde{h}$ is a near homeomorphism. Thus we have a homeomorphism $f: \bar{X}_{h} \rightarrow \widetilde{X}$ by Lemma 3.2. By the invariance of domain, $f\left(X_{h}\right)=X$ since $X_{h}$ is the interior of $\bar{X}_{h}$ by Lemma 3.2. Hence $\left(\bar{X}_{h}, X_{h}\right) \cong(\widetilde{X}, X)$, which completes the proof. 
As seen in Example 2.4, we cannot require $g \in H_{U}(X)$ in the above theorem even if $h$ extends to $\tilde{h} \in H(\tilde{X}) \cap C_{U}(\tilde{X})$.

In the following example, we show that, in general, the hypothesis that $h$ extends to a proper map of $\tilde{X}$ cannot be eliminated from the above theorem. Let $B^{n}$ and $S^{n-1}$ denote the closed unit $n$-ball and the unit $(n-1)$-sphere of $\mathbf{R}^{n}$, i.e., $B^{n}=\left\{x \in \mathbf{R}^{n} \mid\|x\| \leq 1\right\}$ and $S^{n-1}=\left\{x \in \mathbf{R}^{n} \mid\|x\|=1\right\}$. Let $\dot{B}^{n}=B^{n} \backslash S^{n-1}$ be the interior of $B^{n}$.

3.4. Example. For each $n \geq 2$, there is an $h \in H\left(\dot{B}^{n}\right)$ such that $h$ is not conjugate to any uniform homeomorphism. Moreover, $h$ can be chosen $\varepsilon$-close to id for any given $\varepsilon>0$.

Construction. First we consider the case $n=2$ without the $\varepsilon$ condition. For each $i=1,2, \ldots$, let $S_{i}^{1}=\left\{x \in \mathbf{R}^{2} \mid\|x\|=i / i+1\right\}$. Let $\alpha_{2}: \dot{B}^{2} \rightarrow \dot{B}^{2}$ be a homeomorphism such that for each $i, \alpha_{2} \mid S_{i}^{1}=$ id and $\alpha_{2}$ performs a Dehn twist (in any direction) in the annulus region between $S_{i}^{1}$ and $S_{i+1}^{1}$ :

For $n \geq 3$, we define $\alpha_{n}: \dot{B}^{n} \rightarrow \dot{B}^{n}$ by suspending $\alpha_{2}$ as follows:

$$
\alpha_{n}(x)= \begin{cases}x & \text { if }\left(x_{1}, x_{2}\right)=(0,0), \\ \left(\frac{\left\|\left(x_{1}, x_{2}\right)\right\|}{\|x\|} \cdot \alpha_{2}\left(\frac{\|x\|}{\left\|\left(x_{1}, x_{2}\right)\right\|} \cdot\left(x_{1}, x_{2}\right)\right), x_{3}, \ldots, x_{n}\right) & \text { otherwise. }\end{cases}
$$

Let $h=\alpha_{n} \in H\left(\dot{B}^{n}\right)$. We claim that $h$ is not conjugate to any uniform homeomorphism. Suppose on the contrary that $h$ is conjugate to some $g \in$ $H_{U}\left(\dot{B}^{n}\right)$. We write $g=f h f^{-1}$ for some $f \in H\left(\dot{B}^{n}\right)$. Since $g \in H_{U}\left(\dot{B}^{n}\right), g$ extends to a $\tilde{g} \in H\left(B^{n}\right)$. Note $g \mid \bigcup_{i=1}^{\infty} f\left(S_{i}^{n-1}\right)=$ id since $h \mid \bigcup_{i=1}^{\infty} S_{i}^{n-1}=\mathrm{id}$, where $S_{i}^{n-1}=\{x \in \mathbf{R} \mid\|x\|=i /(i+1)\}$. It is easy to see that any neighborhood of each $x \in S^{n-1}$ intersects with $\bigcup_{i=1}^{\infty} f\left(S_{i}^{n-1}\right)$. Therefore $\tilde{g} \mid S^{n-1}=\mathrm{id}$. Then there is a neighborhood $V$ of $S^{n-1}$ such that for each $x \in V$ the line segment between $x$ and $\tilde{g}(x)$ misses the point $f(0)$.

Let $A_{i}$ denote the closed annulus region between $S_{i}^{n-1}$ and $S_{i+1}^{n-1}$, and let

$$
R_{i}=\left\{\left(x_{1}, \ldots, x_{n}\right) \in A_{i} \mid x_{1} \geq 0 \text { and } x_{2}=0\right\}
$$

be an $(n-1)$-cell in $A_{i}$ and $\partial R_{i}$ the boundary of $R_{i}$. Let $r: B^{n} \backslash\{0\} \rightarrow S^{n-1}$ be the radial retraction defined by $r(x)=x /\|x\|$. Then $k=\left(r \mid R_{i}\right)\left(r h \mid R_{i}\right)^{-1}: S^{n-1}$ $\rightarrow S^{n-1}$ is a well-defined null-homotopic map. By choosing $i$ large enough, $f\left(A_{i}\right) \subset V$ so $f\left(R_{i}\right) \cup g f\left(R_{i}\right) \subset V$. Then $f \mid R_{i}$ is homotopic to $g f \mid R_{i}=$ $f h \mid R_{i}$ by the linear-path homotopy in $B^{n} \backslash\{f(0)\}$ fixing $f\left|\partial R_{i}=f h\right| \partial R_{i}$. Thus $r\left|R_{i}=r f^{-1} f\right| R_{i}$ is homotopic to $r h\left|R_{i}=r f^{-1} f h\right| R_{i}$ fixing $r\left|\partial R_{i}=r h\right| \partial R_{i}$. This homotopy induces a homotopy between $k=\left(r \mid R_{i}\right)\left(r h \mid R_{i}\right)^{-1}$ and $\mathrm{id}=$ $\left(r h \mid R_{i}\right)\left(r h \mid R_{i}\right)^{-1}: S^{n-1} \rightarrow S^{n-1}$. This is a contradiction.

To construct $h \quad \varepsilon$-close to id, choose an integer $k>2 \pi / \varepsilon$. In the above construction, we replace the Dehn twist $\alpha_{2}$ by a homeomorphism $\alpha_{2}^{\prime}: \dot{B}^{2} \rightarrow \dot{B}^{2}$ 

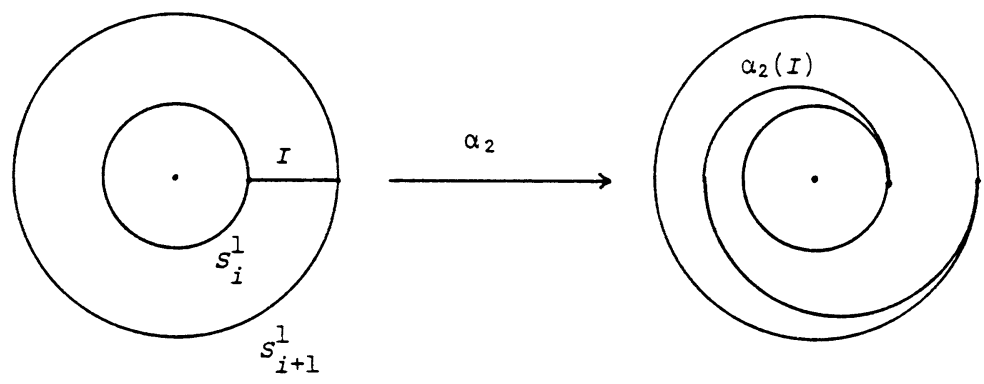

FIgURE 1
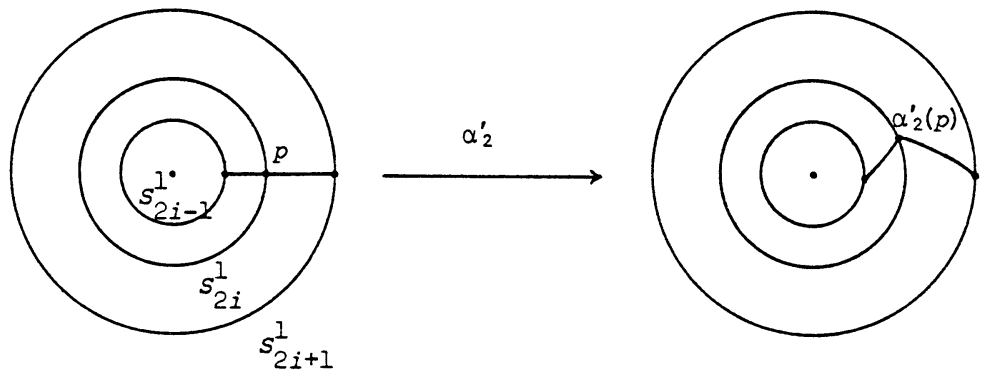

FIGURE 2

such that $\alpha_{2}^{\prime}\left|S_{2 i-1}^{1}=\mathrm{id}, \alpha_{2}^{\prime}\right| S_{2 i}^{1}$ is a $2 \pi / k$-radian rotation. Similarly define $h=\alpha_{2}^{\prime}: \dot{B}^{n} \rightarrow \dot{B}^{n}$ by suspending $\alpha_{2}^{\prime}$. Then $d^{*}(h, \mathrm{id})<\varepsilon$. Similar argument as above shows that $h^{k}$ is not conjugate to any uniform homeomorphism; hence $h$ is not conjugate to any uniform homeomorphism.

As generalizations of (complete) $n$-manifolds, we consider Hilbert cube manifolds ( $Q$-manifolds) and Hilbert manifolds ( $l_{2}$-manifolds). Since $l_{2} \cong s[\mathrm{AB}]$, $l_{2}$-manifolds are also called $s$-manifolds. For a pair $(M, N)$ of $Q$ - (or $l_{2}$-) manifolds, $N$ is collared in $M$ if and only if $N$ is a $Z$-set in $M\left[\mathrm{Ch}_{2}\right.$, Theorem 16.2] or [ $\mathrm{Sa}_{1}$, footnote (1)]. Such a submanifold $N$ is called a $Z$-submanifold of $M$ and it can be considered as a "boundary" of $M$.

3.5. Theorem. Let $X$ be an open submanifold of a $Q$-manifold $\tilde{X}$ with $X^{*}=$ $\widetilde{X} \backslash X$ a Z-submanifold. If $h \in H(X)$ extends to a proper map $\tilde{h}: \widetilde{X} \rightarrow \widetilde{X}$, then $h$ is conjugate to a $g \in H(X)$ which extends to $\tilde{g} \in H(\tilde{X})$.

Proof. As demonstrated in Theorem 3.3, $\tilde{h}: \tilde{X} \rightarrow \tilde{X}$ is a CE-map, hence a near homeomorphism by [Ch ${ }_{2}$, Corollary 43.2$]$. Since $\widetilde{X}$ is locally compact, there is an open cover $\mathscr{U}$ of $\widetilde{X}$ such that for any space $Y$ if a map $g: Y \rightarrow \widetilde{X}$ is $\mathscr{U}$-close to a proper map then $g$ is also proper $\left[\mathrm{Ch}_{2}\right.$, Theorem 4.1, (2)]. From Lemma 3.2, $\bar{\pi}: \bar{X}_{h} \rightarrow \widetilde{X}$ is $\mathscr{U}$-homotopic to a homeomorphism $f: \bar{X}_{h} \rightarrow \widetilde{X}$. Since $\bar{\pi}$ is proper, $f$ is proper homotopic to $\bar{\pi}$. Since $X^{*}$ is a $Z$-set in $\tilde{X}$, $\bar{\pi}^{-1}\left(X^{*}\right)=X_{h}^{*}$ is also a $Z$-set in $\bar{X}_{h}$ by Lemma 3.2. Hence $f\left(X_{h}^{*}\right)$ is a $Z$-set in $\tilde{X}$.

The restriction $h^{*}=\tilde{h} \mid X^{*}: X^{*} \rightarrow X^{*}$ is a CE-map, so again a near homeomorphism. The homeomorphism $q: \bar{X}_{h} \rightarrow \widetilde{X}_{\infty}$ defined in the proof of Lemma 
3.2 restricts to a homeomorphism $q^{*}=q \mid X_{h}^{*}: X_{h}^{*} \rightarrow X_{\infty}^{*}=\lim _{\longleftarrow}\left(X_{i}^{*}, h_{i}^{*}\right)$, where $X_{i}^{*}=X^{*}$ and $h_{i}^{*}=\tilde{h} \mid X^{*}: X_{i}^{*} \rightarrow X_{i-1}^{*}$. By [Cu $\mathrm{Cu}_{1}$, Theorem 3.3], the projection $h_{0 \infty}^{*}: X_{\infty}^{*} \rightarrow X_{0}^{*}=X^{*}$ is a near homeomorphism. Hence $\pi^{*}=\bar{\pi} \mid X_{h}^{*}=h_{0 \infty}^{*} q^{*}: X_{h}^{*} \rightarrow X^{*}$ is a near homeomorphism. Thus we have a homeomorphism $f^{*}: X_{h}^{*} \rightarrow X^{*}$ which is $\mathscr{U}$-homotopic to $\pi^{*}$, hence proper homotopic to $\pi^{*}=\bar{\pi} \mid X_{h}^{*}$. Then $f^{*}$ is proper homotopic to $f \mid X_{h}^{*}$. By the Homeomorphism Extension Theorem [Ch ${ }_{2}$, Theorem 19.4], $f^{*}$ extends to a homeomorphism $\tilde{f}: \bar{X}_{h} \rightarrow \tilde{X}$. Since $\tilde{f}\left(X_{h}^{*}\right)=X^{*}, \tilde{f}\left(X_{h}\right)=X$. Thus $\left(\bar{X}_{h}, X_{h}\right) \cong(\tilde{X}, X)$, which implies the existence of $g$ required in the theorem.

Using the same procedure, we can prove a similar theorem for $l_{2}$-manifolds. In this case, we do not need the assumption that $\tilde{h}$ is proper, but instead we use the $\alpha$-Approximation Theorem [Fe, Theorem 3.4] and the Homeomorphism Extension Theorem [AM].

3.6. Theorem. Let $X$ be an open submanifold of an $l_{2}$-manifold $\tilde{X}$ with $X^{*}=$ $\tilde{X} \backslash X$ a Z-submanifold. If $h \in H(X)$ extends to a map $\tilde{h}: \widetilde{X} \rightarrow \tilde{X}$, then $h$ is conjugate to a $g \in H(X)$ which extends to $\tilde{g} \in H(\tilde{X})$. In particular, each $h \in H(X) \cap C_{U}(X)$ is conjugate to a $g \in H(X)$ which extends to $\tilde{g} \in H(\widetilde{X})$. (Here the metric of $X$ is inherited from the complete metric of $\tilde{X}$.)

Let $M$ be a $Q$-manifold or an $l_{2}$-manifold. We say that a subset $N$ of $M$ is an (f-d) cap set for $M$ if (1) $N=\bigcup_{n=1}^{\infty} N_{n}$, where each $N_{n}$ is a (finitedimensional) compact $Z$-set in $M$ such that $N_{n} \subset N_{n+1}$, and (2) for each $\varepsilon>0$, each integer $m>0$, and each (finite-dimensional) compactum $K$ in $M$, there is an integer $n \geq m$ and an embedding $f: K \rightarrow N_{n}$ such that $f \mid K \cap N_{m}=$ id and $d^{*}(f$, id $)<\varepsilon\left[\mathrm{Ch}_{1}\right]$. When we say that $N$ is an (f-d) cap set for $M$, it means that $N$ is a cap set (or an f-d cap set) for $M$. Let $\sigma$ denote the set of all points in $s$ having at most finitely many nonzero coordinates and let $\Sigma$ denote the set of all points in $s$ having at most finitely many coordinates not in $\left[-\frac{1}{2}, \frac{1}{2}\right]$. Then $\sigma$ is an $\mathrm{f}-\mathrm{d}$ cap set for $s$ and $Q$, and $\Sigma$ is a cap set for $s$ and $Q$. Let $l_{2}^{f}$ denote the linear span of the usual orthonormal basis of $l_{2}$. Then $l_{2}^{f}$ is an $\mathrm{f}$-d cap set for $l_{2}$; hence $\left(l_{2}, l_{2}^{f}\right) \cong(s, \sigma)$ by the topological uniqueness of f-d cap sets [Ch $\mathrm{Ch}_{1}$, Theorem 6.2]. An f-d cap set for an $l_{2}$ - or $Q$-manifold is a $\sigma$-manifold and a cap set for an $l_{2}$ - or $Q$-manifold is a $\Sigma$ manifold [ $\left.\mathrm{Ch}_{1}\right]$. An (f-d) cap set $N$ for a $Q$-manifold $M$ is considered as a "boundary" of $M$ in some sense (see [Cu${ }_{2}$, Introduction]) and $M \backslash N$ is always an $l_{2}$-manifold [ $\left.\mathrm{Ch}_{1}\right]$. On the other hand, a cap set for a $Q$-manifold $M$ is also considered as an "interior" of $M$ in some sense (cf. [BP, Chapter V, Corollary 4.2]). We note that for an (f-d) cap set $N$ for an $l_{2}$ - or $Q$-manifold $M$, the inclusions $N \subset M$ and $M \backslash N \subset M$ are fine homotopy equivalences. (This can be proved by using the Triangulation Theorems, the topological uniqueness of (f-d) cap sets and the fact that the inclusions $\sigma \subset \Sigma \subset s \subset Q$ are fine homotopy equivalences.) 
3.7. Theorem. Let $X$ be an $(f-d)$ cap set for a $Q$-manifold $\tilde{X}$. If $h \in H(X)$ extends to a proper map $\tilde{h}: \widetilde{X} \rightarrow \widetilde{X}$, then $h$ is conjugate to a $g \in H(X)$ which extends to $\tilde{g} \in H(\tilde{X})$.

Proof. Similarly as in Theorem 3.5, $\bar{X}_{h} \cong \tilde{X}$. Since $X$ is map-dense in $\tilde{X}$, $X_{h}$ is also map-dense in $\bar{X}_{h}$ by Lemma 3.2. Recall that $X_{h} \cong X$. By [Sa ${ }_{2}$, Proposition 2.1] (the cap set version is similar), $X_{h}$ is an (f-d) cap set for $\bar{X}_{h}$. By the topological uniqueness of (f-d) cap sets [Ch ${ }_{1}$, Theorem 6.2], $\left(\bar{X}_{h}, X_{h}\right) \cong$ $(\widetilde{X}, X)$. This completes the proof.

The $l_{2}$-version of the above theorem is proved by a different method, in such a case we do not have to impose any condition on $h$ :

3.8. Theorem. Let $X$ be an $(f-d)$ cap set for an $l_{2}$-manifold $\tilde{X}$. Then each $h \in H(X)$ is conjugate to a $g \in H(X)$ which extends to $\tilde{g} \in H(\tilde{X})$.

Proof. Using the Lavrentieff Theorem, there exist $G_{\delta}$-sets $G$ and $G$ in $\tilde{X}$ containing $X$ such that $h$ extends to a homeomorphism $\tilde{h}: G \rightarrow G$. Let $G_{0}=$ $G \cap G^{\prime}$ and $G_{n}=\tilde{h}^{-1}\left(G_{n-1}\right) \cap \tilde{h}\left(G_{n-1}\right)$ for $n=1,2, \ldots$. Then $X^{\prime}=\bigcap_{n=0}^{\infty} G_{n}$ is a $G_{\delta}$-set in $\widetilde{X}$ containing $X$ and $\tilde{h}\left(X^{\prime}\right)=X^{\prime}$. Since $\tilde{X} \backslash X^{\prime}$ is a countable union of $Z$-sets in $\tilde{X}, X^{\prime}$ is homeomorphic to $\tilde{X}$ by [An]. Observe that $X$ is an (f-d) cap set for $X^{\prime}$. By the topological uniqueness of (f-d) cap sets [Ch ${ }_{1}$ ], $\left(X^{\prime}, X\right) \cong(\widetilde{X}, X)$, which implies the theorem.

Next, we will consider the case $X$ is an $l_{2}$-manifold and $\tilde{X}$ is a $Q$-manifold such that $X^{*}=\tilde{X} \backslash X$ is a cap set for $\tilde{X}$.

3.9. Theorem. Let $X$ be an $l_{2}$-manifold contained in a $Q$-manifold $\tilde{X}$ with $X^{*}=\tilde{X} \backslash X$ a cap set for $\tilde{X}$ and let $h \in H(X)$. Suppose there is a cap set $Y$ for $\widetilde{X}$ with $Y \subset X^{*}$ and $h$ extends to a proper map $\tilde{h}: \widetilde{X} \rightarrow \widetilde{X}$ such that $\tilde{h}(Y)=Y$ and $\tilde{h} \mid Y \in H(Y)$. Then $h$ is conjugate to a $g \in H(X)$ which extends to $\tilde{g} \in H(\widetilde{X})$. (Cf. Question 5.1.)

Proof. As seen before, $\bar{X}_{h} \cong \tilde{X}$ and $X_{h} \cong X$. Then $X_{h}$ is a $G_{\delta}$-set in $\bar{X}_{h}$, so $X_{h}^{*}=\bar{X}_{h} \backslash X_{h}$ is an $F_{\sigma}$-set in $\bar{X}_{h}$. Since $X$ is map-dense in $\tilde{X}, X_{h}$ is also mapdense in $\bar{X}_{h}$ by Lemma 3.2. Hence $X_{h}^{*}$ is a countable union of $Z$-sets in $\bar{X}_{h}$. We note that $\bar{Y}_{\hat{h} \mid Y}=\bar{X}_{h}$. As shown in the proof of Theorem 3.7, $Y_{\hat{h} \mid Y}$ is a cap set for $\bar{X}_{h}$. Since $Y_{\hat{h} \mid Y} \subset X_{h}^{*}, X_{h}^{*}$ is also a cap set for $\bar{X}_{h}$ by [Ch ${ }_{1}$, Theorem 6.6]. Again by the topological uniqueness of cap sets, $\left(\bar{X}_{h}, X_{h}^{*}\right) \cong\left(\widetilde{X}, X^{*}\right)$. Hence $\left(\bar{X}_{h}, X_{h}\right) \cong(\tilde{X}, X)$. This completes the proof.

Applying Theorems 3.3, 3.5 and 3.7, we have an answer for Question (A) in case $X$ is a noncomplete manifold admitting a compact manifold completion.

3.10. Corollary. Let $X=(X, d)$ be a metric space. Then each $h \in H(X) \cap$ $C_{U}(X)$ is conjugate to a $g \in H_{U}(X)$ in the following cases:

(1) $X$ is an $n$-manifold $(n \neq 4,5)$ with compact $n$-manifold completion; 
(2) $X$ is a $Q$-manifold with compact $Q$-manifold completion $\tilde{X}$ such that $\tilde{X} \backslash X$ is a $Z$-submanifold;

(3) $X$ is a $\Sigma$ - (or $\sigma$-) manifold with compact $Q$-manifold completion $\tilde{X}$ such that $X$ is an $(f-d)$ cap set for $\tilde{X}$.

\section{STABle homeomorphisms}

In this section we will consider homeomorphisms of the form (or conjugate to) $h \times$ id .

4.1. Lemma. Let $Y$ be a locally compact separable $A R$. Then the one-point compactification $\alpha(Y \times[0,1))=Y \times[0,1) \cup\{\infty\}$ of $Y \times[0,1)$ is an $A R$ and $\{\infty\}$ is a $Z$-set in $\alpha(Y \times[0,1))$.

Proof. Let $\alpha(Y)=Y \cup\{*\}$ denote the one-point compactification of $Y$. We can regard $\alpha(Y \times[0,1))$ as the quotient space

$$
\alpha(Y) \times I /\{*\} \times I \cup \alpha(Y) \times\{1\} .
$$

Let $q: \alpha(Y) \times I \rightarrow \alpha(Y \times[0,1))$ be the identification map. The deformation of $\alpha(Y) \times I$ pushing up along the $I$-coordinates induces a contraction of $\alpha(Y \times$ $[0,1)$ ) onto $\{\infty\}$ fixing $\{\infty\}$. Then $\{\infty\}$ is a strong deformation retract of $\alpha(Y \times[0,1))$. By the result of Kruse and Liebnitz [KL], $\alpha(Y \times[0,1))$ is an AR. For any open cover $\mathscr{U}$ of $\alpha(Y \times[0,1))$, choose $U \in \mathscr{U}$ such that $\infty \in U$. Let $V$ and $W$ be open neighborhoods of $*$ in $\alpha(Y)$ such that $V \times I \subset q^{-1}(U)$ and $\mathrm{cl} W \subset V$ and $k: \alpha(Y) \rightarrow[0,1]$ a Urysohn map such that $k(\mathrm{cl} W)=1$ and $k(\alpha(Y) \backslash V)=0$. Define a map $r: \alpha(Y) \times I \rightarrow \alpha(Y) \times I$ as follows:

$$
r(y, t)= \begin{cases}(y, t) & \text { if } t \geq k(y), \\ (y, k(y)) & \text { if } t<k(y) .\end{cases}
$$

Next choose $0<t_{0}<t_{1}<1$ so that $\alpha(Y) \times\left[t_{0}, 1\right] \subset q^{-1}(U)$. Let $\varphi: Y \times I \rightarrow Y$ be a contraction with $\varphi_{1}(Y)=y_{0}$. Define a map $f: r(\alpha(Y) \times I) \rightarrow Y \times[0,1)$ as follows:

$$
f(y, t)= \begin{cases}\left(y_{0}, t_{1}\right) & \text { if } t_{1} \leq t \leq 1 \\ \left(\varphi\left(y, \frac{t-t_{0}}{t_{1}-t_{0}}\right), t\right) & \text { if } t_{0} \leq t \leq t_{1} \\ (y, t) & \text { if } 0 \leq t \leq t_{0} .\end{cases}
$$

Clearly, $f r: \alpha(Y) \times I \rightarrow Y \times[0,1)$ induces a map $g: \alpha(Y \times[0,1)) \rightarrow Y \times[0,1)$ fixing $(Y \backslash V) \times\left[0, t_{0}\right]$. Since

$$
\alpha(Y \times[0,1)) \backslash(Y \backslash V) \times\left[0, t_{0}\right] \subset U,
$$

$g$ is $\mathscr{U}$-close to id. Then $\{\infty\}$ is a $Z$-set in $\alpha(Y \times[0,1))$.

4.2. Lemma. Let $X$ be an $l_{2}$-manifold. If $X$ is homeomorphic to the product $Y \times Z$ of a locally compact $A R Y$ with $Z$, then $Z$ is homeomorphic to $X$.

Proof. Since $X \approx Y \times Z$ satisfies the discrete approximation property (DAP) $\left[\mathrm{To}_{1}\right]$ and $Y$ is locally compact, $Z$ satisfies DAP. Since $Z$ is a separable 
complete metrizable ANR, it is an $l_{2}$-manifold $\left[\mathrm{To}_{1}\right]$. Thus the projection $p: Y \times Z \rightarrow Z$ is a fine homotopy equivalence (since $Y$ is an AR) between $l_{2}$-manifolds, hence a near homeomorphism [Fe, Theorem 3.4].

4.3. Theorem. Let $X$ be an $l_{2}$-manifold contained in a $Q$-manifold $\tilde{X}$ such that $X^{*}=\tilde{X} \backslash X$ is a cap set for $\tilde{X}$ and let $h \in H(X)$. Suppose there is a homeomorphism $\varphi: X \rightarrow Y \times Z$ of $X$ onto the product of a locally compact $A R$ $Y$ with $Z$ and an $f \in H(Y)$ such that $\varphi h \varphi^{-1}=f \times$ id:

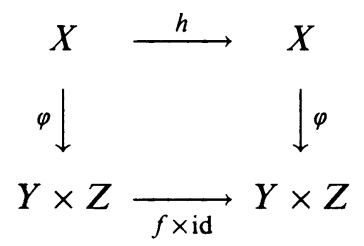

Then $h$ is conjugate to $g \in H(X)$ which extends to $\tilde{g} \in H(\tilde{X})$.

Proof. By Lemma 4.2, we may replace $Z$ by $X$. Since $X \cong X \times[0,1)$, we have a homeomorphism $\psi: Y \times X \rightarrow Y \times[0,1) \times X$ with the commutative diagram:

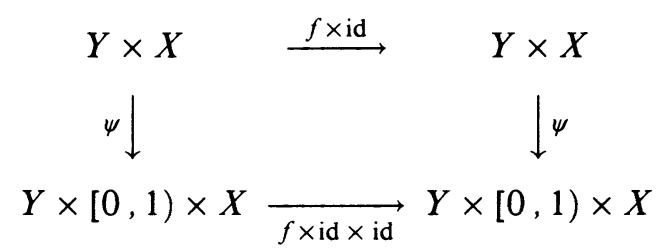

Let $Y^{\prime}=\alpha(Y \times[0,1))=Y \times[0,1) \cup\{\infty\}$ be the one-point compactification of $Y \times[0,1)$. Then $f \times$ id $\in H(Y \times[0,1))$ extends to $f^{\prime} \in H\left(Y^{\prime}\right)$ with $f^{\prime}(\infty)=\infty$. Clearly $f^{\prime} \times$ id $\in H\left(Y^{\prime} \times X\right)$ extends to $f^{\prime} \times$ id $\in H\left(Y^{\prime} \times \widetilde{X}\right)$. Since $Y^{\prime}$ is a compact AR by Lemma 4.1 and $\widetilde{X}$ is a $Q$-manifold, we have $Y^{\prime} \times \tilde{X} \cong \widetilde{X}$. It is easy to see that $Y^{\prime} \times X^{*}$ is a cap set for $Y^{\prime} \times \widetilde{X}$. Since $\{\infty\} \times \tilde{X}$ is a $Z$-set in $Y^{\prime} \times \tilde{X}$ by Lemma 4.1 ,

$$
Y^{\prime} \times \tilde{X} \backslash Y \times[0,1) \times X=Y^{\prime} \times X^{*} \cup\{\infty\} \times \tilde{X}
$$

is a cap set for $Y^{\prime} \times \tilde{X}\left[\mathrm{Ch}_{1}\right.$, Theorem 6.6]. By the topological uniqueness of cap sets,

$$
\left(Y^{\prime} \times \tilde{X}, Y \times[0,1) \times X\right) \cong(\tilde{X}, X) .
$$

This implies the theorem.

By the same proof, we have the following theorem.

4.4. Theorem. Let $X$ be an $(f-d)$ cap set for a $Q$-manifold $\tilde{X}$ and $h \in$ $H(X)$. Suppose there is a homeomorphism $\varphi: X \rightarrow Y \times X$ of $X$ onto a product of a (finite-dimensional) locally compact $A R Y$ with $X$ and an $f \in H(Y)$ such 
that $\varphi h \varphi^{-1}=f \times \mathrm{id}$ :

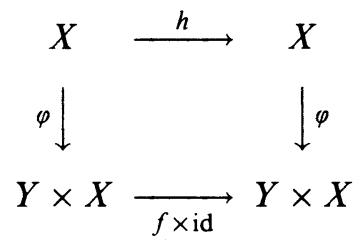

Then $h$ is conjugate to a $g \in H(X)$ which extends to $\tilde{g} \in H(\tilde{X})$.

Remark. By examples of Henderson and Walsh [HW], Lemma 4.2 is not true in case $X$ is a $\Sigma$ - (or $\sigma$-) manifold. We do not know whether $X$ of $Y \times X$ in the above theorem can be replaced by another space $Z$.

4.5. Theorem. Let $X$ be an $l_{2}$-manifold contained in a Q-manifold $\tilde{X}$ such that $\tilde{X} \backslash X$ is a cap set for $\tilde{X}$. If $h \in H(X)$ extends to a proper map $\tilde{h}: \tilde{X} \rightarrow \tilde{X}$, then $h \times \mathrm{id} \in H(X \times s)$ is conjugate to a $g \in H(X \times s)$ which extends to $\tilde{g} \in H(\tilde{X} \times Q)$.

Proof. Let $Y_{0}$ be a cap set for $X$. Then $Y=\bigcup_{n \in \mathbf{Z}} h^{n}\left(Y_{0}\right)$ is also a cap set for $\tilde{X}\left[\mathrm{Ch}_{1}\right.$, Theorems 6.3 and 6.6]. By [ $\mathrm{Ch}_{1}$, Theorem 6.8], $Y \times(Q \backslash s)$ is a cap set for $\widetilde{X} \times Q$. Since $h \times$ id $\in H(X \times s)$ extends to a proper map $\tilde{h} \times$ id: $\tilde{X} \times Q \rightarrow \widetilde{X} \times Q$ such that $\tilde{h} \times$ id $\mid Y \times(Q \backslash s) \in H(Y \times(Q \backslash s))$, the result follows from Theorem 3.9.

As a consequence of Theorems 4.3 and 4.5, we have an answer for Question (5) in $[\mathrm{AB}]([\mathrm{AK}, \mathrm{CSQ} 4])$ :

4.6. Corollary. Let $h: s \rightarrow s$ be a homeomorphism of the pseudo-interior of the Hilbert cube $Q$ onto itself. In the following cases, $h$ is conjugate to a $g \in H_{U}(s)$ (which extends to $\tilde{g} \in H(Q)$ ).

(1) There is a homeomorphism $\varphi: s \rightarrow Y \times Z$ of $s$ onto the product of a locally compact $A R Y$ with another space $Z$ and an $f \in H(Y)$ such that $\varphi h \varphi^{-1}=f \times \mathrm{id}:$

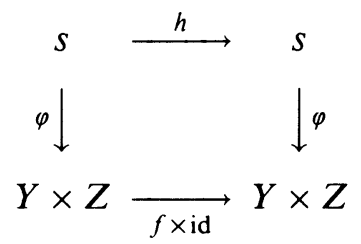

(2) There is a homeomorphism $\varphi: s \rightarrow s \times s$ and an $f \in H(s) \cap C_{U}(s)$ such that $\varphi h \varphi^{-1}=f \times$ id :

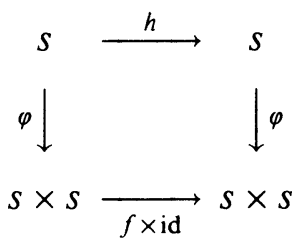


Remark. The $\sigma$ - or $\Sigma$-version of the above also holds. For (2), we have already a better result in Corollary 3.10 .

Let $i^{n}=I^{n} \backslash \partial I^{n}$ denote the interior of $I^{n}$. The following is a finitedimensional version of the above corollary.

4.7. Corollary. Let $h: i^{n} \rightarrow I^{n}(n>1)$ be a homeomorphism which is conjugate to the form $f \times \mathrm{id}$, where $f \in H\left(i^{n-1}\right)$. Then $h$ is conjugate to a $g \in H_{U}\left(\dot{I}^{n}\right)$ (which extends to $\tilde{g} \in H\left(I^{n}\right)$ ).

Proof. This follows from the fact

$$
\left(I^{n}, i^{n}\right) \cong\left(I^{n} / \partial I^{n-1} \times I \cup I^{n-1} \times\{1\}, i^{n}\right) .
$$

Remark. Each homeomorphism $h: i \rightarrow \dot{I}$ is clearly a uniform homeomorphism.

\section{OPEN QUESTIONS}

5.1. If $X$ is an $l_{2}$-manifold with the compact $Q$-manifold completion $\tilde{X}$ such that $\tilde{X} \backslash X$ is a cap set, is every $h \in H(X) \cap C_{U}(X)$ conjugate to a $g \in H_{U}(X)$ ? 5.2. Is every homeomorphism of $s$ onto $s$ conjugate to a uniform homeomorphism of $s$ onto $s$ ?

5.3. We do not know the answer if $s$ in the above question is replaced by $X$, where $X=l_{2}, \mathbf{R}^{n}, n \geq 2$ or a Banach space.

5.4. Let $h: \dot{B}^{2} \rightarrow \dot{B}^{2}$ be the homeomorphism constructed in Example 3.4. Given $X=\dot{B}^{2} \times \dot{B}^{2} \times \cdots$ the usual product metric, is the homeomorphism $h \times h \times \cdots$ conjugate to a uniform homeomorphism on $X$ ? If the answer is "no", then it is a counterexample to 5.2.

Added in proof. Recently J. van Mill has constructed a counterexample to Question 5.2 (i.e. [AB, Question (5), AK, CSQ4]) in his paper: A homeomorphism on $s$ not conjugate to an extendable homeomorphism.

\section{REFERENCES}

[An] R. D. Anderson, Strongly negligible sets in Fréchet manifolds, Bull. Amer. Math. Soc. 75 (1969), 64-67.

[AB] R. D. Anderson and R. H. Bing, A complete elementary proof that Hilbert space is homeomorphic to the countable infinite product of lines, Bull. Amer. Math. Soc. 74 (1968), 771-792.

[AK] R. D. Anderson and N. Kroonenberg, Open problems in infinite-dimensional topology, Topological Structures, P. C. Baayen, ed., MCT 52, Math. Centrum, Amsterdam, 1974, pp. 141175.

[AM] R. D. Anderson and J. D. McCharen, On extending homeomorphisms to Fréchet manifolds, Proc. Amer. Math. Soc. 25 (1970), 283-289.

[BP] C. Bessaga and A. Pełczyński, Selected topics in infinite-dimensional topology, MM 58, Polish Sci. Publ., Warsaw, 1975.

[Br] M. Brown, Some applications of an approximation theorem for inverse limits, Proc. Amer. Math. Soc. 11 (1960), 478-483.

$\left[\mathrm{Ch}_{1}\right]$ T. A. Chapman, Dense sigma-compact subsets of infinite-dimensional manifolds, Trans. Amer. Math. Soc. 154 (1971), 399-426. 
[Ch $\left.\mathrm{Ch}_{2}\right]$, Lectures on Hilbert cube manifolds, CBMS Regional Conf. Ser. in Math., no. 28, Amer. Math. Soc., Providence, R.I., 1976.

$\left[\mathrm{Cu}_{1}\right]$ D. W. Curtis, Near homeomorphisms and fine homotopy equivalences, unpublished manuscript.

$\left[\mathrm{Cu}_{2}\right]$ _- Boundary sets in the Hilbert cube, Topology Appl. 20 (1985), 201-221.

[Fe] S. Ferry, The homeomorphism group of a compact Hilbert cube manifold is an ANR, Ann. of Math. (2) 106 (1977), 101-119.

[HW] J. P. Henderson and J. J. Walsh, Examples of cell-like decompositions of the infinite-dimensional manifolds $\sigma$ and $\Sigma$, Topology Appl. 16 (1983), 143-154.

[KL] A. H. Kruse and P. W. Liebnitz, An application of a family homotopy extension theorem to ANR spaces, Pacific J. Math. 16 (1966), 331-336.

[Sa 1 ] K. Sakai, An embedding theorem of infinite-dimensional manifold pairs in the model space, Fund. Math. 100 (1978), 83-87.

[Sa $\left.{ }_{2}\right]$ _ $A$ Q-manifold local-compactification of a metric combinatorial $\infty$-manifold, Proc. Amer. Math. Soc. 100 (1987), 775-780.

[Si] L. Siebenmann, Approximating cellular maps by homeomorphisms, Topology 11 (1972), 271294.

[To 1 ] H. Torunczyk, Characterizing Hilbert space topology, Fund. Math. 111 (1981), 247-262.

[ $\left.\mathrm{To}_{2}\right]$ ] A correction of two papers concerning Hilbert manifolds, Fund. Math. 125 (1985), 89-93.

Institute of Mathematics, University of Tsukuba, Tsukuba, 305 JaPan 93106

Department of Mathematics, University of California, Santa Barbara, California 